\title{
Development of in situ Imaging Capabilities in SEM and Their Applications
}

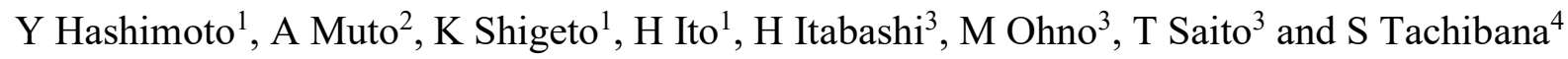 \\ 1. Application Development Dept., Hitachi High-Technologies Corporation, Kawasaki, Japan. \\ 2. Nanotechnology System Division, Hitachi High Technologies America, Inc., MD, USA. \\ 3. Electron Microscope Systems Design $1^{\text {st }}$ Dept., Hitachi High-Technologies Corporation, Hitachinaka, \\ Japan. \\ 4. Marketing Dept., Hitachi High-Technologies Corporation, Tokyo, Japan.
}

Scanning electron microscope (SEM)s are widely used to observe fine structures of various types of materials, and recently demands of in situ observation with the SEM have been increasing to elucidate dynamics in heating or tensile studies. Demands to monitor transition of microstructures with measurements of breaking strength or ultimate tensile strength of industrial materials, such as metals and polymers, are increasing in research and development of these materials [1].

Although in situ tensile SEM observation was realized by using a tensile sub stage, two technical difficulties have been recognized in in situ SEM imaging for metals and polymers. One is slow response of photo diode type backscattered electron detector(PD-BSED)s for metals. The PD-BSED has been suitable for metallographic study due to its sensitivity for crystal channeling contrast imaging, but generally it has not for in situ study which requires fast scan live image refresh rate. The other is low image resolution in ultra-low voltage imaging condition for polymers. Imaging at ultra-low voltage below $500 \mathrm{~V}$ is necessary to avoid charge-up artifact and beam damage for polymers in SEM observation. Applying bias voltage to the sample stage is one of the techniques to enhance image resolution at ultralow voltage condition, however it is inapplicable to the tensile testing due to the configuration of the tensile sub stage.

In this study, developments of in situ imaging capabilities in SEM to resolve these two technical difficulties are reported and some applications are demonstrated. A schottky type FE-SEM (Hitachi SU7000) was used. Its newly designed objective lens enables to enhance image resolution without stage bias voltage application. Figure 1 shows the SU7000 and schematic illustration of its signal detection system. Five imaging detectors, including in-column secondary electron (SE) detector: UD, and backscattered electron (BSE) detector: MD, are adopted in the microscope. All detectors including an energy dispersive x-ray spectroscopy (EDS) detector can work effectively in the same working distance (WD). The newly designed PD-BSED realized imaging with fast scan rate with less streak effect by optimizing applied bias voltage and circuit configuration.

Tensile behavior of an aluminum foil was examined. A Gatan Microtest300 was used for tensile testing in the SEM chamber. Figure 2 shows frame-by-frame images of a movie which recorded real time SEM image of the specimen with the new PD-BSED while tensile force was applied. Dynamic behaviors of crystal grains which were deformed to be finally torn were clearly recorded with less streak effect. In situ applications of various types of materials will be also reported. 
Reference:

[1] M Kubo et al., ISIJ Int. 56 (4) (2016), p. 669.

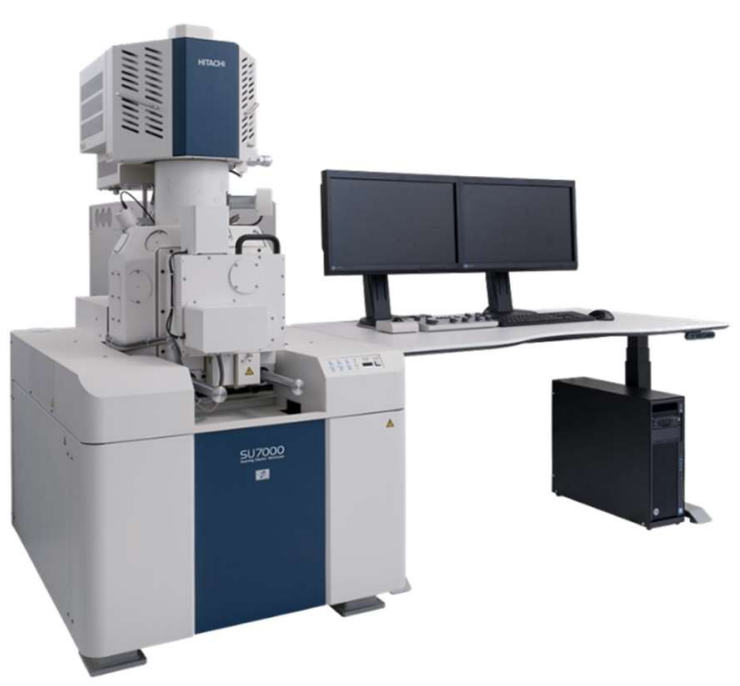

(a)

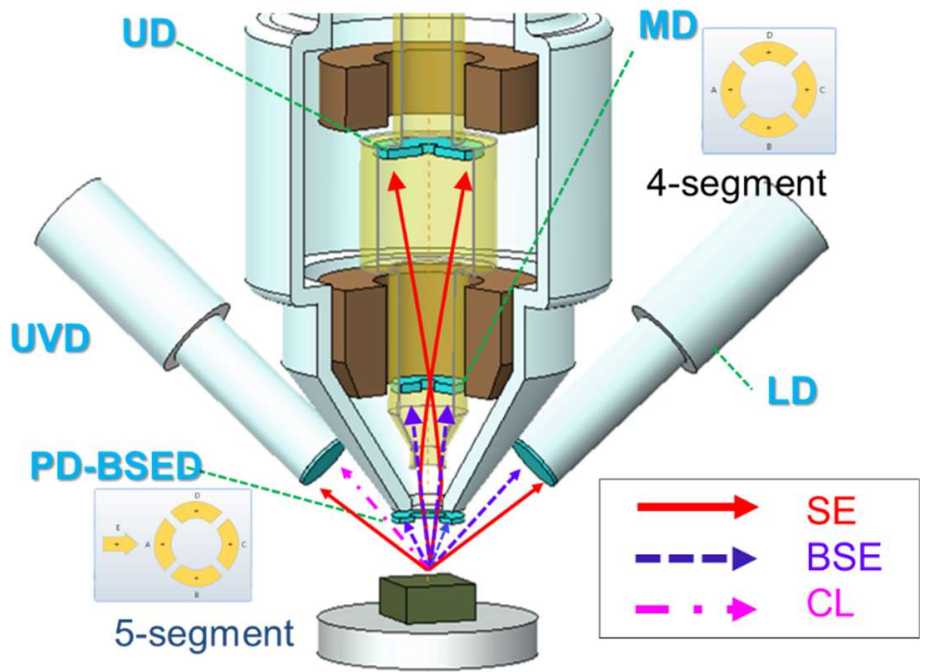

(b)

Figure 1. (a) Hitachi SU7000 FE-SEM and (b) Schematic illustration of signal detection system.
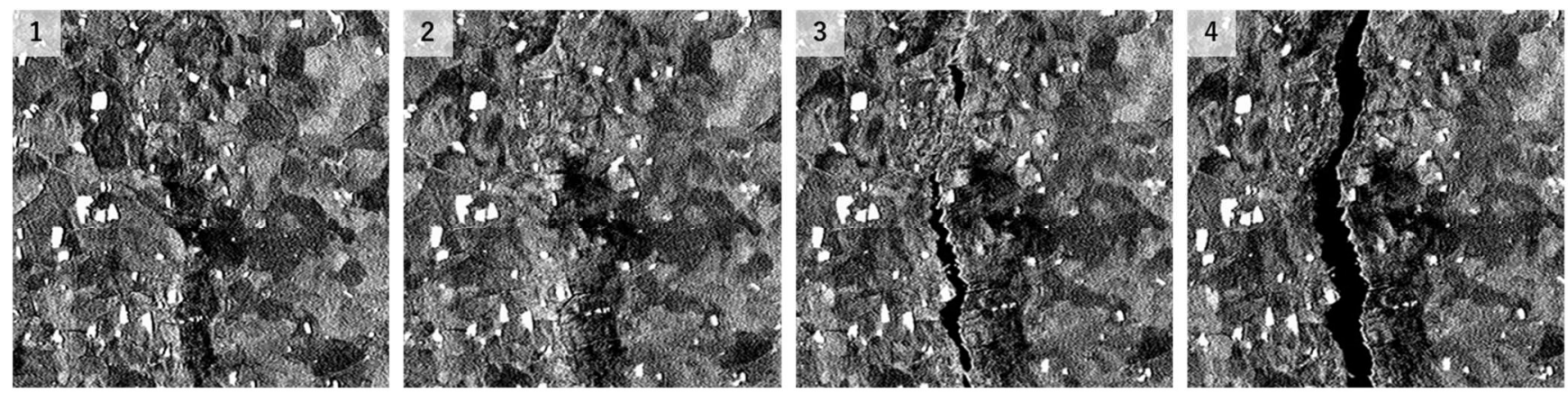

$10 \mu \mathrm{m}$

Figure 2. Frame-by-frame images of a movie which recorded real time SEM images with new PD-BSED while tensile force was applied. (Acceleration voltage: $5 \mathrm{kV}$ ) 